\title{
Constant Stress Creep Tester Using the Servo Divider*
}

\author{
By Takeo Murata**
}

\begin{abstract}
In order to examine the transient creep, especially its initial stage in detail, a new constant stress creep tester has been developed. The load necessary to maintain the stress constant can be obtained by an automatic control system with a servo divider, which calculates the equation, as $W=W_{0} l_{0} /\left(l_{0}+x\right)$, where $W_{0}$ is the initial load, $l_{0}$ is the initial gauge length and $x$ is the elongation of the specimen. The specimen is stretched by the dead weight of water which is controlled in accordance with the calculated value. The system has the advantages of giving the load under a constant loading condition and keeping the stress constant against various disturbances. The maximum load is $100 \mathrm{~kg}$ with precision of $\pm 0.3 \mathrm{~kg}$, and the deviation of the load from the calculated value is within $1 \mathrm{~kg}$. A new precise temperature controller which stabilizes the temperature within $\pm 0.1^{\circ} \mathrm{C}$ is also proposed. The tester makes it possible to obtain the load-elongation curves before the creep tests and creep curves up to the strain of $10 \%$. On the basis of the fact that polycrystalline $\mathrm{Fe}-19 \mathrm{Cr}$ alloy exhibits the incubation phenomenon within the strain of $0.1 \%$, it is suggested that the phenomenon will be found below such a small strain in some alloy systems which have not exhibited the phenomenon thus far.
\end{abstract}

(Received July 23, 1974)

\section{Introduction}

Various methods for keeping the stress constant have been proposed ${ }^{(1) \sim(4)}$ to obtain creep characteristics, especially the transient creep properties precisely. Andrade adjusted the load by allowing the weight to sink into a liquid as the specimen stretched ${ }^{(1)}$. As well known, constant stress can be also obtained by using the Andrade-Chalmers lever arm of which the ratio changes in accordance with the creep elongation $^{(2)}$. Thrower was successful in changing the ratio by using a servo mechanism ${ }^{(3)}$. In these methods, however, there were some problems such as precise machining of the loading systems and the elimination of disturbances which make the stress uncertain.

In order to settle these problems, an automatic control system with a servo divider has been introduced. Under the condition that the specimen elongates uniformly along the gauge length and the density of the specimen is kept constant, the load necessary to maintain the stress constant can be expressed by the equation

* This paper was published partly in J. Japan Inst. Metals, 33 (1969), 1359.

** The Research Institute for Iron, Steel and Other Metals, Tohoku University, Sendai 980, Japan.

Trans. JIM

$$
W=W_{0} l_{0} /\left(l_{0}+x\right),
$$

where $W_{0}$ is the initial load, $l_{0}$ is the initial gauge length and $x$ is the elongation of the specimen. $W$ is calculated continuously by the servo divider into which a signal proportional to $\left(l_{0}+x\right)$ is fed. The load acting on the specimen is measured by a load cell and is automatically controlled in accordance with the calculated value of $W$. The loading system can give accurate stress on the specimen in a vacuum chamber against various disturbances such as the force which arises when the bellows extend. The system has also an advantage of giving a load under the condition of a constant loading rate. Accordingly, the tester has sufficient capability necessary to examine the behavior of the transient creep, especially of its initial stage in detail. The tester has operated in a stable manner and satisfactorily during the last six years. In this report, the outline of the tester and some experimental results are described.

\section{Equipment description}

\section{(1) Loading system}

Figure 1 shows the block diagram of the automatic control system for keeping the stress constant. The load is applied to the specimen by means of a lever arm (not shown in the figure) having the ratio of 20:1 and the dead

1975 Vol. 16 
Takeo Murata

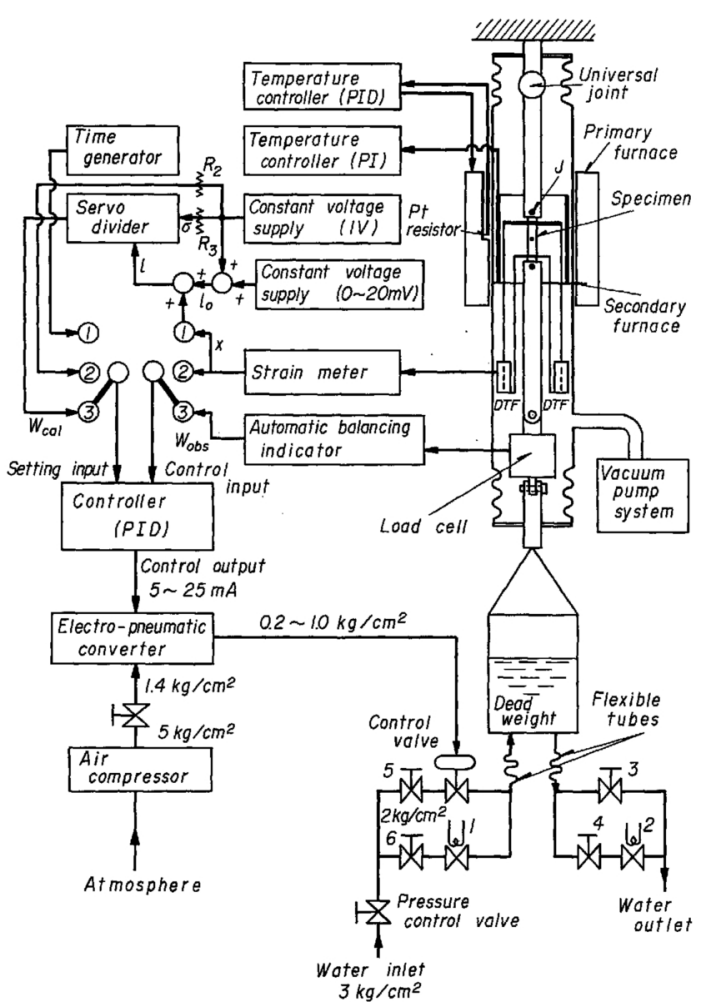

Fig. 1 Block diagram of the automatic control system. Double switch position 1: tensile tests at constant strain rate, 2: stress relaxation tests, 3: constant stress creep tests.

weight of water. In order to adjust the weight of water, draining from the water vessel and supplying it with water are carried out simultaneously. When the constant stress creep tests are performed, the amount of water supplied decreases as the elongation increases. By this means, the load can be varied smoothly without any mechanical vibration. The weight of water is adjusted by the PID controller so as to minimize the difference between the force acting on the specimen and the calculated value. As shown in Fig. 1, the gauge length, 50-55 $\mathrm{mm}$, is converted to voltages ranging from 1 to $1.1 \mathrm{~V}$ by adding the output voltages of the voltage supply I, II and the strain meter, where the output voltages I and II are proportional to the initial gauge length and the thermal expansion of the specimen, respectively. Figure 2 shows the electric circuit of the servo divider ${ }^{(5)}$, which calculates the equation, $W_{\text {cal }}=k \sigma / 1$, where $k$ is a constant depending on the voltage supplied

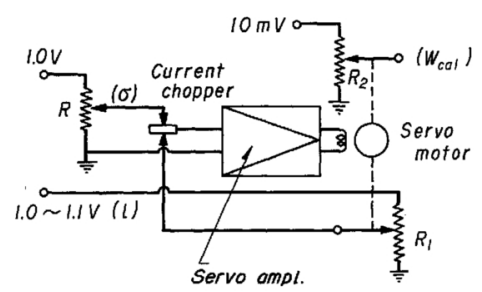

Fig. 2 Electric circuit for the servo divider.

to $R_{2}$. The applied stress can be varied by adjusting $R_{3}$. The precision of the divider is as high as $0.5 \%$ of the maximum quotient, $10 \mathrm{mV}$, under the condition that the value of the denominator varies from 1.0 to $1.1 \mathrm{~V}$ and the value of the numerator is adjusted at the voltages ranging from 0 to $1 \mathrm{~V}$. Precision is obtained when the five-revolution potentiometer having a minimum scale of $1 / 500$ is used. The divider has operated in a very stable manner and has been insensitive to fluctuations of the line voltage and noises. At the beginning of the creep tests, it is necessary to increase the load up to $W_{0}$ under a constant condition. In order to achieve this, the output of the time generator is connected with the setting input of the PID controller instead of $W_{\text {cal }}$ until the load attains $W_{0}$. Accordingly, the load-elongation curves can be obtained at the load up to $W_{0}$ under a constant loading rate condition. The tester is also designed for tensile tests and stress relaxation tests as shown in Fig. 1.

\section{(2) Temperature control system}

The primary furnace having the capacity of $2.4 \mathrm{~kW}$ is installed outside the vacuum vessel, and its temperature is controlled to about $\pm 1{ }^{\circ} \mathrm{C}$ by the PID controller. The fluctuation of the temperature can be minimized by the secondary furnace which is put in the vessel. Figure 3 shows the electric circuit of the precise temperature controller for the secondary furnace. The furnace is assembled by winding a platinum wire, $0.3 \mathrm{~mm}$ in diameter and with a length of $15 \mathrm{~m}$, on a half fused alumina cylinder having the outer diameter of $50 \mathrm{~mm}$ and a length of $150 \mathrm{~mm}$. $R_{p t}$ stands for the electrical resistance of the furnace and takes the values of 20 and 80 $\Omega$ at 20 and $800^{\circ} \mathrm{C}$, respectively. One can balance the bridge which consists of $R_{p t}$, 


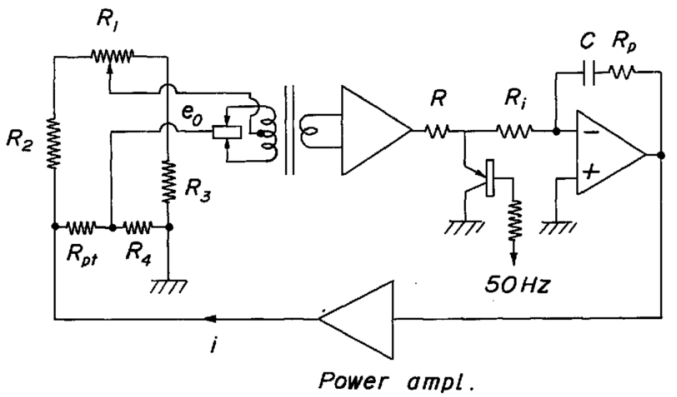

Fig. 3 Electric circuit for the precise temperature controller.

$R_{1}(=100-200 \Omega), R_{2}(=1500-4800 \Omega), R_{3}(=$ $500 \Omega$ ) and $R_{4}(=10 \Omega)$ by adjusting the brush of $R_{1}$ to a certain point. As only the value of $R_{p t}$ depends on the temperature, the error voltage of $e_{0}$ is nearly proportional to the deviation from the set-up temperature. In order to decrease the deviation, the current, $i$, is controlled by the integrator, which minimizes the steady-state offset temperature. The proportional band of $2^{\circ} \mathrm{C}$ has been obtained by using the $\mathrm{AC}$ amplifier having a gain of $60 \mathrm{~dB}$, and putting the constants as $R_{i}=5 \mathrm{k} \Omega, R_{p}=100 \mathrm{k} \Omega$ and $\mathrm{C}=75 \mu F$. The maximum output voltage and current of the power amplifier are $50 \mathrm{~V}$ and $1 \mathrm{~A}$, respectively. As the secondary furnace acts also as the temperature detector, the control system exhibits a rapid response when various disturbances take place. The temperature can be controlled to within $\pm 0.1^{\circ} \mathrm{C}$ in a stable manner by this method.

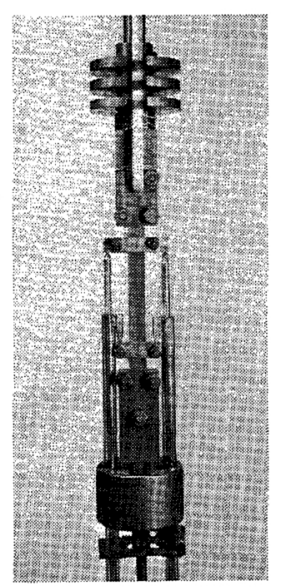

Photo. 1 Method for detecting the elongation of the specimen.

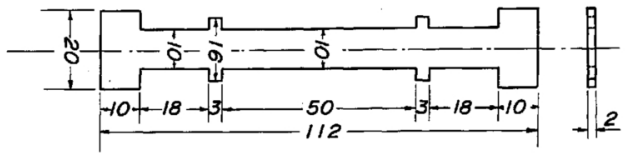

Fig. 4 The shape and size of the specimen.

\section{(3) Method for detecting the creep elonga- tion}

The creep elongation is detected at the end of the quartz glass tube by two differential transformers as shown in Photo. 1. As the tubes are pushed against the extension fittings which are attached to the projections of the specimen (see Fig. 4), the elongation can be detected accurately even if thermal expansion or reduction of the cross sectional area occurs and the projection moves perpendicularly to the specimen axis.

\section{Some Experimental Results}

The tester makes it possible not only to measure the amount of the instantaneous elongation but also to know whether or not the creep stress is larger than the yield stress, as shown in Fig. 5. As shown also in the figure, polycrystalline iron exhibits a normal transient creep behavior, however, polycrystalline $\mathrm{Fe}-$ $19 \mathrm{Cr}$ alloy shows an incubation phenomenon below the strain of $10^{-3}$, which is much smaller than the values observed in $\mathrm{Fe}-3.5 \mathrm{Si}^{(6)}, \mathrm{Cu}-$ 16 at $\% \mathrm{Al}^{(7)}, \alpha$-brass $^{(8)}$ and nickel base super alloy $^{(9)}$. It is expected that such a phenomenon will be found in the other alloy systems at a very small strain region. This phenomenon will play an important role in the transient creep region and bring about a better understanding of the creep of metals.

\section{Summary}

(1) The load necessary to maintain the stress constant can be obtained by an automatic control system with a servo divider, which calculates the equation, as $W=W_{0} l_{0} /\left(l_{0}+x\right)$. This system has the advantages of giving the load under a constant loading condition and keeping the stress constant against various disturbances. The maximum load is $100 \mathrm{~kg}$ 


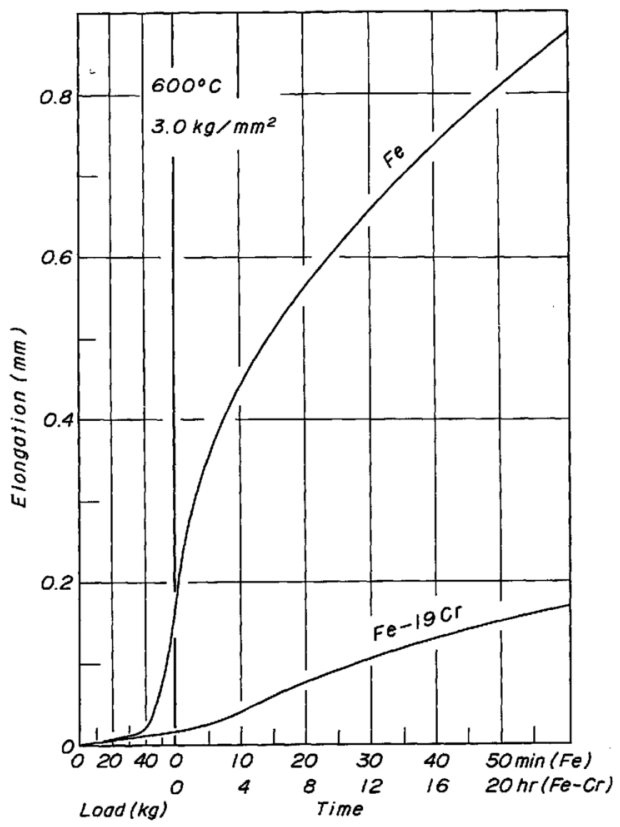

Fig. 5 Load-elongation curves and transient creep curves of $\mathrm{Fe}$ and $\mathrm{Fe}-19 \mathrm{Cr}$ alloy.

with a precision of $\pm 0.3 \mathrm{~kg}$, and the deviation of the load from the calculated value is within $1 \mathrm{~kg}$.

(2) The temperature can be controlled to within $\pm 0.1^{\circ} \mathrm{C}$ by using the platinum furnace which also acts as the temperature detector, and the integrator.

(3) The tester makes it possible to obtain the load-elongation curves before the creep tests and creep curves up to the strain of $10 \%$. On the basis of the fact that $\mathrm{Fe}-19 \mathrm{Cr}$ alloy exhibits the incubation phenomenon within the strain of $0.1 \%$, it is suggested that the phe- nomenon will be found below such a small strain in some alloy systems which have not exhibited the phenomenon thus far.

\section{Acknowledgments}

The author wishes to thank Emeritus Prof. Y. Imai for his kind guidance and Prof. $\mathbf{R}$. Matsuo for his useful advice on the automatic control system. He is also indebted to Mr. T. Nakazawa for his discussion on the design for the tester, Mr. H. Yamaguchi and Mr. Y. Endo for their drafting, and the staffs at the factory attached to the Research Institute for their precise machining of the parts.

The author would like to express his thanks to Mr. K. Kawakami and Mr. H. Kimura for their assistance in this experiment.

\section{REFERENCES}

(1) E. N. da C. Andrade: Proc. Roy. Soc., A84 (1910), 1.

(2) E. N. da C. Andrade and B. Chalmers: Proc. Roy. Soc., A138 (1932), 348.

(3) E. N. Thrower: J. Sci. Instr., 29 (1952), 91.

(4) A. J. Kennedy: J. Sci. Instr., 29 (1952), 40.

(5) for instance, T. Miura and T. Fujimaki: Electronic Computers, Ed. by $\mathrm{H}$. Yamashita, Volume for Analog Computers, The Ohm Company, (1959).

(6) M. Pahutová, T. Hostinský and J. Čadek: Acta Met., 20 (1972), 693.

(7) T. Hasegawa, Y. Ikeuchi and S. Karashima: Metal Sci. J., 6 (1972), 78.

(8) H. L. Burghoff and C. H. Mathewson: Metals Technology, 8 (1941). Technical Publication, No. 1288.

(9) G. A. Webster and B. J. Piearcey: Metal Sci. J., 1 (1967), 97. 